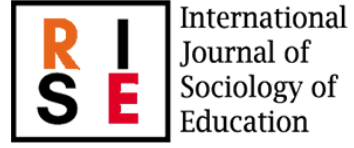

Journal of Sociology of Education

\section{Hipatia Press}

www.hipatiapress.com

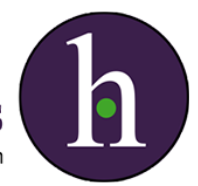

Instructions for authors, subscriptions and further details:

http://rise.hipatiapress.com

\title{
Resistencias y Adhesiones Escolares: La Importancia del Origen Social
}

Ona Valls ${ }^{1}$

1) Universitat Autònoma de Barcelona, Spain

Date of publication: June $25^{\text {th }}, 2021$

Edition period: June 2021-October 2021

To cite this article: Valls, O. (2021). Resistencias y Adhesiones Escolares: La Importancia del Origen Social. International Journal of Sociology of Education, 10(2), 143-170. http://doi.org/10.17583/rise.2021.6660

To link this article: http://dx.doi.org/10.17583/rise.2021.6660

\section{PLEASE SCROLL DOWN FOR ARTICLE}

The terms and conditions of use are related to the Open Journal System and to Creative Commons Attribution License (CC-BY) 


\section{Resistance and Adhesion at School: The Importance of Social Origin}

Ona Valls

Universitat Autònoma de Barcelona

(Received: 17 September 2020; Accepted: 3 December 2020; Published: 25 June 2021)

\section{Abstract}

Different currents and traditions have studied student attitudes at school, and more specifically much of the research has focused on school resistance. The study is based on an explanatory design of sequential mixed methods that consists of collecting quantitative data and then qualitative data to explain on the quantitative results. In this way, this article shows results from the qualitative phase based on the analysis of semi-structured interviews. It analyses the reasons why students have attitudes of adhesion or school resistance at the end of Compulsory Secondary Education (ESO) and how this influences their post-compulsory educational pathways. The results show an intersectionality when the attitude of the pupils at the end of compulsory education is analysed. Important differences are observed by class, origin and gender. The important role played by teachers and schools, their practices and the support they provide to students is highlighted.

Keywords: school resistance, school adhesion, educational trajectory, student's attitude, students' transition 


\section{Resistencias y Adhesiones \\ Escolares: La Importancia del Origen Social}

Ona Valls

Universitat Autònoma de Barcelona

(Recibido: 17 Septiembre 2020; Aceptado: 3 Diciembre 2020; Publicado: 25 Junio 2021)

\section{Resumen}

Distintas corrientes y tradiciones han estudiado la actitud del alumnado en la escuela, y gran parte de las investigaciones se han centrado en las resistencias escolares. A partir de un diseño explicativo de métodos mixtos secuenciales, que consiste en recoger primero datos cuantitativos y después datos cualitativos para explicar o elaborar los resultados cuantitativos, se presentan resultados de la fase cualitativa a partir del análisis de entrevistas semiestructuradas. Se analizan los motivos por los que el alumnado tiene actitudes de adhesión o de resistencia al final de la Educación Secundaria Obligatoria (ESO) y cómo influye en sus itinerarios educativos posteriores. Los resultados muestran una interseccionalidad a la hora de analizar la actitud del alumnado, se observan importantes diferencias por razón de clase, origen y sexo. Se pone de manifiesto el importante papel que juega el profesorado y el centro de enseñanza, sus prácticas y el acompañamiento que hacen del alumnado.

Palabras clave: resistencia escolar, adhesión escolar, trayectoria educativa, actitud del alumno, transición de estudiantes 


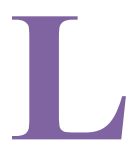

as actitudes del alumnado en la escuela han sido ampliamente estudiadas desde distintas perspectivas y aproximaciones. Una de las actitudes más estudiada ha sido la resistencia escolar dado que se ha vinculado con el abandono educativo.

De este modo, se realizaron análisis cuantitativos previos y este artículo pretende dar respuesta a algunos de esos resultados. Se trata de un diseño secuencial explicativo que reúne la información secuencialmente en dos fases, primero cuantitativa y después cualitativa con el fin de explicar y profundizar en los resultados cuantitativos, haciendo énfasis en los casos atípicos o extremos (Creswell y Guetterman, 2019).

Más concretamente el objetivo de este artículo es profundizar en como la experiencia escolar y las características sociodemográficas de los y las jóvenes los llevó a desarrollar actitudes de rechazo y resistencia o, por el contrario, de adhesión a la escuela y si eso ha tenido una influencia sobre sus trayectorias formativas postobligatorias. Para ello, en el primer apartado se presentan las principales aportaciones teóricas respecto a las actitudes del alumnado en la escuela, más concretamente las de adhesión y resistencia. En el segundo apartado se explica la metodología utilizada, la muestra y las hipótesis del artículo. Finalmente, se presentan los resultados empíricos de las entrevistas analizadas y las conclusiones.

\section{Marco Teórico}

En la literatura encontramos numerosas clasificaciones de las actitudes del alumnado en la escuela. Algunas hacen referencia al alumnado anti-escuela y pro-escuela, o conformista y no conformista (Hargreaves, 1967; Macleod, 1995; Rodríguez, 2015; Suárez-Orozco y Suárez-Orozco, 2008). También hay aproximaciones más amplias que adaptan el esquema de Merton (1938) para hablar de la aceptación del éxito académico como objetivo a perseguir en la escuela (Woods, 1987). O la definida por Bernstein (1977) que hace referencia a la identificación instrumental y expresiva con la escuela. Entendiendo la identificación instrumental como el aprendizaje formal que debe realizarse o como el valor instrumental que se da a la escuela considerándola un medio para conseguir un fin (Barbeta y Termes, 2014; Duru-Bellat y Van Zanten, 1999; Subirats y Alegre, 2006). Por otro lado, la identificación expresiva se 


\section{Valls - Resistencias y Adhesiones Escolares}

vincula al ámbito normativo de la institución. Se basa en la aceptación de las normas escolares, es decir, la respuesta que da el alumnado al comportamiento requerido por la escuela (Aggleton, 1987; Alegre y Benito, 2012; Bonal et al., 2010; Woods, 1987). En ambos ejes, el expresivo y el instrumental, las definiciones utilizadas hacen referencia a actitudes y creencias -interés intelectual, aceptación de las normas o vinculación, entre otros-.

Por otro lado, encontramos otras perspectivas enfocadas hacia el interés intelectual y cultural del alumnado (Dubet, 1992; Aggleton, 1987; Everhart, 1983), señalando que el desarrollo de tal interés produciría una ruptura emocional e intelectual con los orígenes de algunos grupos sociales (DuruBellat y Van Zanten, 1999). Otros autores se acercan de forma más amplia al concepto hablando de distanciamiento respecto de los valores y normas (Subirats y Alegre, 2006), de la vinculación escolar (Barbeta y Termes, 2014) o de la opinión que tienen los estudiantes de la escuela (Griffin, 1985).

La resistencia escolar ha sido la actitud más ampliamente estudiada. Encontramos un primer modelo de alienación según Bernstein (1977), o de inhibición según Merton (1938), que se define por su falta de identificación instrumental y expresiva. Así, puede darse una actitud de inhibición o también de contraposición con el discurso escolar. Esto último se aproxima a las actitudes de resistencia de la clase trabajadora que describe Willis (1977), la más habitualmente encontrada en la literatura (Abraham, 1989; Anyon, 1981; Barbeta y Termes, 2014; Bonal et al., 2010; Brown, 1987; Dubet, 1992). En referencia a la teoría de la resistencia que realizó Willis (1977) en los años 70', hay que remarcar que fue en un contexto de cultura industrial obrera diferente del actual en el que hay una terciarización de la economía con un importante desarrollo de las nuevas tecnologías.

Sin embargo, otros estudios encuentran perfiles de chicos de clase media y alta (Aggleton, 1987; Anyon, 1981; Valls, 2020) pero los chicos de clase media y alta que encuentra Aggleton (1987), a pesar de presentar actitudes anti escuela, no muestran un discurso articulado de oposición. Y lo mismo ocurre con los estudiantes de escuela de élite de Anyon (1981) que muestran rechazo a la escuela pero adoptan la apariencia física, modales y lenguaje de la clase trabajadora. Por otro lado, encontramos estudios que apuntan a la existencia de subculturas juveniles de oposición, de chicos y chicas de clase media, que recurren a elementos alternativos de carácter artístico (Abraham, 
1989). De este modo, estudios recientes (Valls, 2020) muestran que hay actitudes, como las de resistencia o inhibición, que ya no se asocian principalmente con la clase trabajadora. Finalmente, cabe destacar otras formas de resistencia exclusivamente femenina que se basan en un proyecto de familia, maternidad y trabajo reproductivo, adoptando formas de comportarse que hacen referencia a la condición femenina adulta (Griffin, 1985; McRobbie, 1978).

De este modo, es posible encontrar estudiantes que responden a un modelo poco esperado, como los estudiantes de clase media resistentes o de clase trabajadora identificados con la escuela. Y no sólo en estudios actuales (Valls, 2020), sino también entre las monografías clásicas (Aggleton, 1987; Anyon, 1981). Además, para medir la actitud del alumnado muchos autores han puesto el foco en los comportamientos observables del alumnado, como el grado de conflictividad, el esfuerzo dedicado al estudio (Alegre y Benito, 2012; Dubet, 1992) o los resultados académicos (Bonal et al., 2010). Estas aproximaciones se acercan a la del engagement escolar (Archambault et al., 2009; Wang et al., 2019) que predice en cierto grado la repetición (Yang et al., 2018), el abandono escolar o la continuidad educativa (Ream y Rumberger, 2008) y la expulsión (Toldson et al., 2013).

\section{Factores Que Intervienen}

Cuando se analiza por qué el alumnado adopta una determinada actitud hay que tener distintos factores en cuenta. Por un lado, influyen los factores individuales como la edad dado que hay un efecto de la maduración personal que puede modificar la identificación con la escuela (Duru-Bellat y Van Zanten, 1999; Pianta et al., 2012). También pueden producirse acontecimientos críticos que modifiquen la actitud del alumnado como, por ejemplo, problemas de salud, procesos de retorno migratorio, etc. (García y Razeto, 2019; Subirats y Alegre, 2006). Por otro lado, el rendimiento escolar obtenido tiene una influencia sobre la actitud, unos malos resultados pueden influir en el rechazo escolar modificando las actitudes del alumnado (Bonal et al., 2010; Brown, 1987; Dubet, 1992; Galland, 1988; Rodríguez, 2015). En cambio, unos buenos resultados educativos favorecen la identificación con la escuela (Brown, 1987; Duru-Bellat y Van Zanten, 1999; Everhart, 1983). 


\section{Valls - Resistencias y Adhesiones Escolares}

También el contexto institucional y las prácticas escolares juegan un papel importante, tanto su cultura, formas de acogida, prácticas, organización en niveles de aprendizaje, capacidad de adaptación, profesorado u orientación son elementos clave para entender las actitudes del alumnado en la escuela (Ball, 1981; Bonal et al., 2010). De este modo, en referencia a los procesos de agrupación escolar, estos afectan a las experiencias del alumnado y también a sus identidades académicas y sociales con un importante impacto en la construcción de la vinculación escolar (Pàmies y Castejón, 2015). Y como se ha mencionado, en ese proceso es importante el papel que juega el profesorado (Duru-Bellat y Van Zanten, 1999).

Las adscripciones de género, clase y etnia también pueden ser transformadas en actitudes continuas con la socialización o neutralizadas por las experiencias de los adolescentes en los espacios sociales que se mueven. Estos son factores, según expone Bonal y otros (2003) que posicionan a los individuos en el mapa social y pueden condicionar la forma que tienen de construir o transgredir sus actitudes frente a la escuela. De este modo, en relación con la clase trabajadora se evidencia una escasa identificación expresiva con la institución escolar dado que estudiantes y familias priorizan el valor instrumental de la inversión educativa como mecanismo de inserción laboral satisfactoria (Bonal et al., 2003). Por otro lado, la relación entre socialización familiar y éxito escolar se entiende como la relación entre el conjunto de disposiciones, esquemas de razonamiento, formas de relacionarse con la autoridad que imperan en la familia y en la escuela. Así, en la medida que los tipos de relación en los dos ámbitos coinciden se favorecerá la adaptación al universo escolar o aparecerán fuentes divergentes (MartínCriado et al., 2000). Pero no hay que confundir clase social y familia, tal y como expone Carabaña (1993) ya que se pueden encontrar distintos estilos de socialización dentro de cada clase social. Por último, hay que tener en cuenta también la importancia de las expectativas familiares respecto a los estudios $\mathrm{y}$ los recursos de los que disponen.

Respecto al género este está presente en la construcción de las disposiciones sociales y educativas. Dada la socialización diferenciada entre chicos y chicas (Bonal et al., 2003) o como un mecanismo de compensación frente a las peores perspectivas futuras de inserción profesional las chicas tienen una mayor predisposición al estudio y una mejor adaptación al entorno 
escolar. En cambio, los chicos tienen una mayor predisposición a la disrupción escolar (Gambetta, 1987) dado que tienden más a identificarse instrumentalmente con la escuela y las chicas expresivamente (Bonal et al., 2003). Hay que tener en cuenta que características como el sexo o el origen, elementos que son evidentes para el profesorado, pueden tener un efecto en la construcción de las expectativas que tienen los propios profesores del alumnado. Como expone Rist (1991) los profesores esperan menos del alumnado de clase baja que del de clase media. De este modo, las expectativas del profesorado o el efecto Pigmalión (Rist, 1991) también pueden tener influencia en la construcción de la identificación con la escuela.

En referencia al origen o la etnia, hay que tener en cuenta que el alumnado de determinadas procedencias y sus familias ven la inversión educativa como estrategia de movilidad social, interpretando así la escuela únicamente des de coordenadas instrumentales (Bonal et al., 2003). Hay diferentes estudios que muestran evidencias de una mayor ambición educativa por parte del alumnado inmigrantes con actitudes más positivas frente a la escuela que los estudiantes autóctonos (Rodríguez, 2015).

Finalmente, el contexto social tiene influencia en la consideración del papel económico y social de la escuela. Un contexto de más oportunidades de trabajo cualificado percibidas daría lugar a una mayor valoración instrumental de la escuela (Valls, 2020).

\section{Metodología}

En el marco del International Study of City Youth (ISCY) se realizaron un total de 46 entrevistas a jóvenes, 24 mujeres y 22 hombres de entre 15 y 16 años, que cursaban $4^{\circ}$ de la ESO en el curso 2013/2014 en un instituto de la ciudad de Barcelona. Esta fase cualitativa del proyecto se realizó para complementar un estudio panel de 4 años a una muestra representativa de jóvenes de la ciudad de Barcelona. La primera ola del proyecto se realizó cuando los y las jóvenes cursaban $4^{\circ}$ de la ESO o nacidos en 1998 y que se encontraban en cursos inferiores por estar repitiendo algún curso. Se hizo un seguimiento con encuestas durante los tres años posteriores hasta el curso 2016/2017. La segunda fase del proyecto se basó en entrevistar a algunos y algunas estudiantes que participaron en el panel para poder profundizar en los 


\section{Valls - Resistencias y Adhesiones Escolares}

resultados cuantitativos obtenidos. Como se ha mencionado, se trata de un diseño explicativo de métodos mixtos secuenciales que consiste en recoger primero datos cuantitativos y después datos cualitativos para explicar o elaborar los resultados cuantitativos. De este modo, los datos y resultados cuantitativos muestran un panorama general del problema de investigación y mediante los datos cualitativos se amplia y explica este marco general. Esto permite dar mayor detalle o seguimiento de casos atípicos o extremos (Creswell y Guetterman 2019), un diseño analítico poco explorado para analizar las actitudes del alumnado en la escuela. En este tipo de diseños la fase cuantitativa tiene un peso mayor y guía a la cualitativa, y es importante considerar la forma de seleccionar la muestra cualitativa del estudio. Es recomendable que los individuos de la fase cualitativa hayan participado en la primera fase para poder representar de forma más detallada sus experiencias y percepciones (Hamui-Sutton, 2013) tal y como se realiza en el presente estudio. De este modo, la muestra de las entrevistas se seleccionó intencionalmente dentro de la muestra de jóvenes del panel de acuerdo a los criterios de heterogeneidad, accesibilidad y voluntariedad. Se tuvieron en cuenta las características sociodemográficas -sexo, origen y el nivel formativo familiar- y el itinerario formativo en la educación postobligatoria.

Se realizaron entrevistas biográficas semiestructuradas, cuando tenían entre 17 y 18 años, dos años más tarde de realizar la primera encuesta, con el objetivo de explorar las elecciones educativas que realizaron después de la escuela obligatoria. Las entrevistas realizadas fueron estandarizadas no programadas (Valles, 1999) y se realizaron de forma presencial o telefónica, con una duración aproximada de 45 minutos.

De la totalidad de las 46 entrevistas en este artículo se muestran los resultados de 23 de las entrevistas correspondientes a los y las jóvenes que tenían actitudes más polarizadas en $4^{\circ}$ de la ESO. Es decir, a los y las jóvenes con actitudes de resistencia escolar (Figura 2) - 11 entrevistas - y con actitudes de adhesión escolar (Figura 1) - 12 entrevistas-. El análisis se ha realizado con el software Atlas.ti.

1. A continuación, se muestran los perfiles entrevistados en función del itinerario formativo que han seguido después de la ESO y de su clase social. La clase social se basa en la categoría profesional más elevada de los progenitores a partir de ISCO-08 y posteriormente siguiendo el 
esquema EGP (Erikson y Goldthorpe 1992). A partir de aquí las categorías de EGP se han agrupado del siguiente modo:

a) Empresarios y profesionales (I + II).

b) Pequeños propietarios y trabajadores no manuales (III + IV).

c) Trabajadores manuales (V+VI + VII).

Cabe especificar que las actitudes de adhesión o resistencia analizadas se dieron en el último curso de la ESO dado que el objetivo de este artículo es analizar el efecto de las actitudes al final de la ESO sobre los itinerarios formativos posteriores.

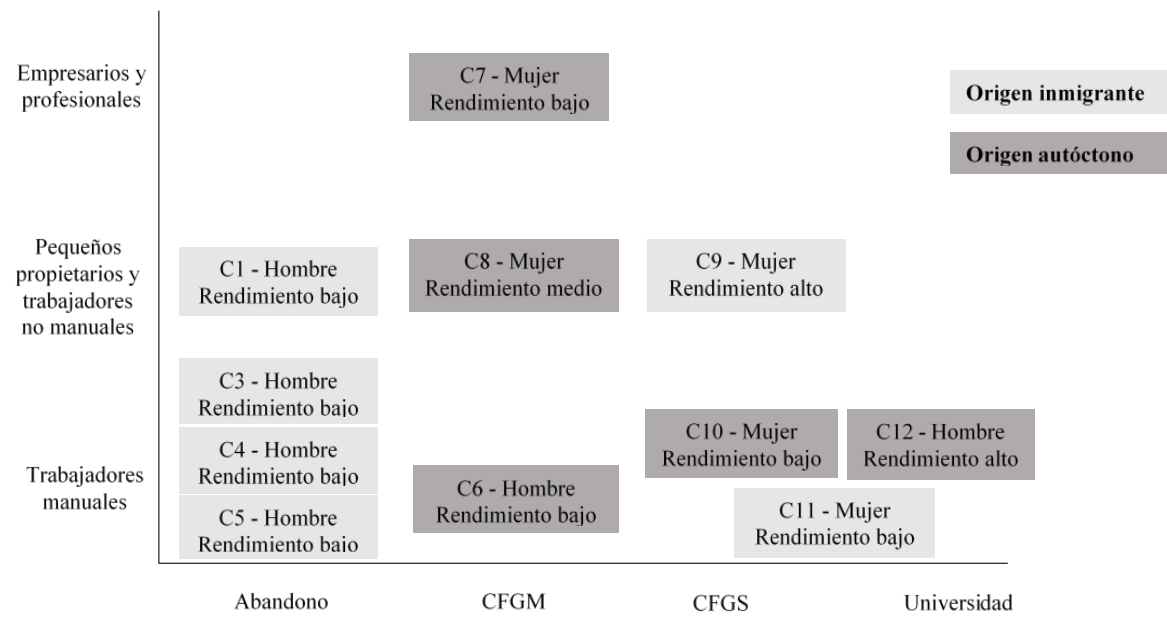

Figura 1. Entrevistados adheridos en $4^{\circ}$ de la ESO en función del origen social y del itinerario formativo posterior 


\section{Valls - Resistencias y Adhesiones Escolares}

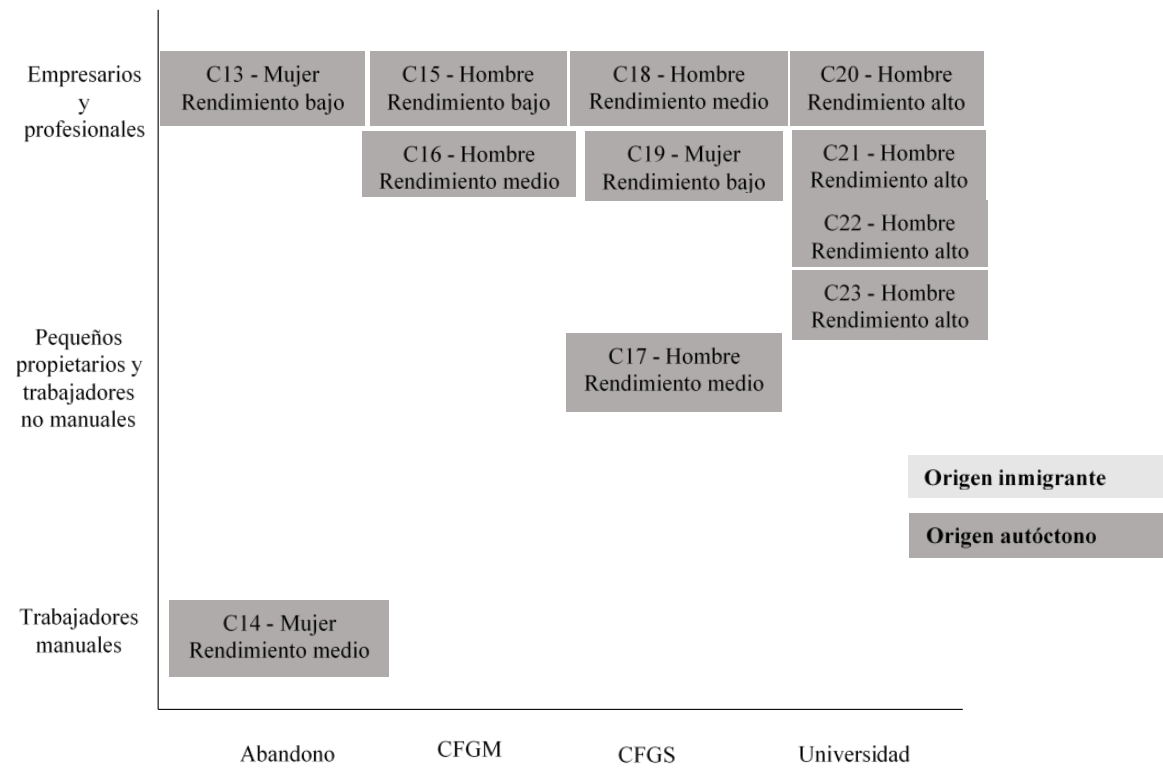

Figura 2. Entrevistados resistentes en $4^{\circ}$ de la ESO en función del origen social y del itinerario formativo posterior

\section{Hipótesis}

Dado que los y las jóvenes entrevistados forman parte de un estudio panel $(n=2056$ alumnos), se han realizado análisis cuantitativos previos que han aportado mucha información. Los objetivos del estudio cuantitativo eran: (1) crear una tipología de actitudes del alumnado al final de la ESO siguiendo la tipología clásica de Bernstein (1977). (2) Describir como las variables sociodemográficas inciden en la adscripción a una determinada actitud. (3) Explorar el efecto de las actitudes sobre el itinerario educativo posterior. De este modo, conocíamos de antemano el nivel de identificación instrumental y expresiva con la escuela que tenían estos jóvenes en $4^{\circ}$ de la ESO. A partir de aquí, los objetivos del estudio cualitativo son: (1) ahondar en el discurso de los y las jóvenes sobre porque consideran que tenían esa actitud de adhesión o resistencia desde un enfoque interseccional. (2) Conocer si la actitud que 
tenían los influyó a la hora de continuar con sus estudios o de abandonarlos. De este modo, las hipótesis del presente artículo se plantean en base a los resultados cuantitativos obtenidos previamente:

H.1. Los y las jóvenes de origen inmigrante tienen, en mayor medida que los autóctonos, actitudes de adhesión escolar dado que creen en la educación como forma de ascenso social -identificación instrumental-.

H.2. Las chicas de origen inmigrante desarrollan actitudes de adhesión escolar dado que ven la escuela como una oportunidad de mejora de futuro identificación instrumental- $\mathrm{y}$, por otro lado, interpretan la escuela como un lugar de oportunidad y aceptación -identificación expresiva-.

H.3. Los chicos de clase media con actitudes de resistencia creen que la escuela secundaria no es importante porque dan por supuesto que independientemente de cómo les vaya en la secundaria irán a la universidad.

\section{Resultados}

Entre las personas jóvenes entrevistadas encontramos grupos de estudiantes que responden a modelos poco esperados, lo que se corresponde con los resultados cuantitativos previos realizados en el proyecto ISCY Barcelona. Por un lado, como ya se observaba en los análisis cuantitativos, encontramos jóvenes adheridos de origen inmigrante y clase trabajadora. Y, en cambio, los jóvenes resistentes destacan por ser de origen autóctono y clase media. De hecho, entre los y las jóvenes entrevistados no hay ningún estudiante resistente de origen inmigrante.

\section{Adhesiones Escolares}

Jóvenes adheridos autóctonos. Los jóvenes autóctonos entrevistados con actitudes de adhesión han tenido malas experiencias con sus pares o problemas de rendimiento y finalmente han elegido continuar con estudios de formación profesional después de la educación secundaria obligatoria (ESO).

En el caso de los y las jóvenes autóctonos con un mal rendimiento destacan distintas situaciones de dificultad, pero en todos los casos se sintieron apoyados por la escuela y el profesorado. Por un lado, encontramos experiencias negativas en relación al grupo de pares como son casos de 


\section{Valls - Resistencias y Adhesiones Escolares}

bullying:

Pues empezaré por los negativos porque he tenido más negativos que positivos. Los negativos son que mis compañeros no eran muy amigables que dijéramos. Me hacían bullying. Me hicieron bullying desde primero de la ESO hasta tercero. Repetí curso voluntariamente para sacarme a esas personas de encima y empezar de nuevo. Y aquí fue cuando empecé a ir bien. Compañeros nuevos, vida nueva y ya está. En ese momento no vi ninguna otra solución que no fuera repetir - C7. Chica. Empresarios y profesionales - CFGM

Por otro lado, aparece el tema de la adaptación curricular, que en general es valorado positivamente por la ayuda recibida por parte del profesorado:

Pues bien, un poquillo complicado, pero bueno, lo hice bien. Me ayudaron un poco también porqué digamos que no hice lo que hacen todos (...) Pues porque digamos que a mí no me gustaba mucho estudiar y cuando ya pase a primero pues ya me vieron que no me gustaba estudiar. Mis padres también y bueno, hablaron con los profesores y eso y me hicieron esta ayuda para poder sacarme la ESO con un 5 aunque sea y para luego tener un futuro y poder trabajar... y eso - C8. Chica. Pequeños propietarios y trabajadores no manuales - CFGM

Jóvenes adheridos de origen inmigrante. Respecto a los y las jóvenes adheridos de origen inmigrante entrevistados, los chicos abandonan los estudios a pesar de haber tenido una buena experiencia en la educación obligatoria y, en cambio, las chicas continúan con estudios postobligatorios. De este modo, los y las jóvenes destacan un sentimiento de superación durante la escuela secundaria:

Porque por lo menos obtuve la ESO. La ESO es importante, por lo menos obtener la ESO - C4. Chico. Trabajadores manuales - Abandono

Cuando llegue en la ESO, en secundaria, pues he tenido más para aprenderlo y lo conseguí. Conseguí aprender bien español y catalán. $\mathrm{Y}$ las cosas que no entendía pues lo he superado todo (...) $\mathrm{Y}$ en un 
momento dado me gustaba estudiar, me gustaba estar estudiando, por motivos que sale las cosas bien, entendía, las cosas que tenía muchas dudas he conseguido hacerlo yo mismo, y lo conseguía - C2. Chico. Trabajadores manuales - Abandono

Por otro lado, están agradecidos con el profesorado por haberlos ayudado en su proceso de aprendizaje, en su llegada al país de acogida y a conseguir el título de la Educación secundaria obligatoria:

La verdad que no me quejo de los profesores que tenía porque, tenía unos profesores y ...que la verdad que pensé que iba a pasar mucho tiempo que me iba a olvidar de muchas cosas y la verdad que no... los consejos que me han dado eran muy importantes (...) Porque hay gente que no obtuvo la ESO y teniendo buenos profesores porque en eso no me voy a quejar - C4. Chico. Trabajadores manuales - Abandono

Bien, me han ayudado mucho, me han ayudado en las cosas que no podía... Me han ayudado. Si no entendía pues me explicaban de otra manera para entenderlo más mejor y lo entendía bien - C2. Chico. Trabajadores manuales - Abandono

La mayoría destacan recuerdos positivos de su escolaridad, pero son relevantes distintas situaciones familiares complejas, procesos migratorios, entre otros:

Bueno, positivos siempre... tengo muchos recuerdos, lo que pasa...Lo que pasa he tenido un problema, que ha fallecido mi hermana y mi madre tiene que ir a Bangladesh y he dejado de estudiar, no hacia los deberes y he suspendido, he repetido (...) siempre pensaba cosas negativas siempre tenía un miedo por dentro, no está mi madre al lado, y nadie que yo cuando iba a casa, nadie me preguntaba de dónde vienes y tal porque estaba mi padre y mi padre trabajaba, y venía a la 1 de la noche a casa y yo a esa hora estaba durmiendo - C3. Chico. Trabajadores manuales - Abandono

Prácticamente me costó un poco, el cambio de país de origen a país 


\section{Valls - Resistencias y Adhesiones Escolares}

extranjero, cambio de cole y amigos, me costó un poco... Pero la ayuda de los profesores y compañeros me ayudo - C1. Chico. Pequeños propietarios y trabajadores no manuales - Abandono

A pesar de valorar positivamente su paso por la escuela secundaria y de que a muchos les hubiera gustado seguir estudiando destacan que no pudieron por dificultades económicas, familiares y personales o de rendimiento. De este modo, muchos de estos jóvenes no pueden continuar sus estudios.

Bueno, pues me hubiese gustado seguir estudiando. ¿Seguido no? O sea, poder haber hecho bachillerato, sí, yo siempre he dicho que en bachillerato no, no me veo, y es que no me gusta, lo considero para una persona que se aplique a los estudios al máximo, y quiera ir directamente a la universidad hasta llegar a tener un máster. - C4. Chico. Trabajadores manuales - Abandono

Saqué la ESO. Bueno, no pude seguir bachillerato porque tenía problemas económicos, familiares - C2. Chico. Trabajadores manuales - Abandono

A mí me gustaba estudiar, porque yo en la primaria, desde primero de la primaria estoy estudiando en Barcelona. Era un chaval que siempre tenía ganas de estudiar. Aun cuando me ven en la calle mis profesores de primaria me preguntan como estas, estas estudiando, no, y me avergüenza decir no y digo sí - C3. Chico. Trabajadores manuales - Abandono

Otro elemento que emerge como justificación del abandono educativo es el hecho de llegar a Barcelona de adolescente como una dificultad añadida:

Emm... prácticamente porque o sea las materias eran un poco, o sea eso ya fue en cuarto, porque me costaba un poco el hecho del inglés y las ciencias, y las mate que estaban un poco avanzadas. Ya al suspenderla no podía pasar el curso y tenía que dejarlo sí o sí. Porque ya no podía repetirlo tampoco quería repetirlo. Ya después de eso pedí un cupo en PI para terminar la media - C1. Chico. Pequeños propietarios y trabajadores no manuales - Abandono 
Finalmente, los y las jóvenes adheridos de origen inmigrante, a parte de sentirse agradecidos con el profesorado también destacan positivamente la buena relación con sus pares:

Positivos pues los amigos también puede ser - C11. Chica. Trabajadores manuales - CFGS y Universidad

Positivos de la ESO los profesores, compañeros, era todo más fácil, no me costaba tanto. Negativo casi ninguno- C9. Chica. Pequeños propietarios y trabajadores no manuales - CFGS

Estos resultados nos llevan a confirmar nuestra primera hipótesis dado que los y las estudiantes adheridos de origen inmigrante entrevistados ven la educación como forma de ascenso social, y se identifican instrumentalmente con la escuela. Pero también se identifican expresivamente con esta y se sienten bien en la escuela. A pesar de esto, son exclusivamente las chicas entrevistadas las que continúan con estudios postobligatorios. Esto no nos permite confirmar nuestra segunda hipótesis dado que no se observan discursos diferenciados entre chicos y chicas de origen inmigrante a la hora de explicar sus actitudes de adhesión. A pesar de esto, se observa que el hecho de desarrollar actitudes de adhesión beneficia positivamente a las chicas inmigrantes si tenemos en cuenta sus itinerarios formativos.

\section{Resistencias Escolares}

Respecto a los jóvenes resistentes hay un tema recurrente que son los problemas con el profesorado. Pero dentro de estos jóvenes observamos importantes diferencias según clase social y género. Se observa como en el caso del alumnado entrevistado son las chicas a las que las actitudes de resistencia les trae peores resultados educativos si observamos su itinerario. Por un lado, destaca la diferencia entre chicos y chicas resistentes de clase media. Los chicos de clase media entrevistados todos continúan su formación con estudios postobligatorios, la mayoría sigue el itinerario académico de bachillerato y universidad. En cambio, en el caso de las chicas resistentes de 


\section{Valls - Resistencias y Adhesiones Escolares}

clase media entrevistadas o abandonan sus estudios o realizan estudios profesionales después de la ESO.

Chicos resistentes. Entre los chicos de clase media hay que destacar que cuatro realizan un itinerario académico y tres un itinerario profesional como estrategia para ir a la universidad. Y la diferencia entre los que optan por un itinerario u otro es su rendimiento académico.

De este modo, se observa que el profesorado juega un papel muy importante. Estos jóvenes, por un lado, destacan como motivo de su desinterés y resistencia durante la secundaria el hecho de tener mala relación con el profesorado:

Los profesores horribles, lo hicieron muy mal (...) Bueno yo creo que no sabían gestionar bien. Había alumnos que no estábamos en nuestro mejor momento o simplemente teníamos dieciséis años y no estábamos por lo que teníamos que estar. Con dieciséis años sacarme de clase o meterme la bronca delante de todos por hablar en clase o cosas así, creo que no se deberían hacer y se repetían semana sí semana también - C20. Chico. Empresarios y profesionales - Universidad

Los estudiantes de clase media o media-baja que continúan hacia estudios profesionales también apuntan problemas o desmotivación respecto al profesorado:

Yo creo que el profesorado en tema de estudios obligatorios es bastante flojo. En aspectos yo creo que deberían dar más oportunidades a gente joven que viene con ganas realmente y no a gente que parece que vaya allí a pasar el rato. Y realmente profesores que haya tenido en la ESO a mí me han gustado dos o tres y eran de las asignaturas que a mí me gustaban como biología o educación física. Otros profesores que he tenido no me han gustado porque no se les ve con ganas, con ímpetu, con innovación en las clases - C16. Chico. Empresarios y profesionales - CFGM

Incluso uno de los entrevistados destaca como el hecho de cambiar de centro modificó su experiencia y actitud en la escuela. De este modo emerge 
el importante papel del profesorado, de su ayuda, acompañamiento y expectativas:

El cambio de centro a otro instituto me resultó bastante agradable porque los profesores te trataban más de tú a tú, se preocupaban más por tus intereses y estaba bien que, por ejemplo, el tutor me ayudó a que me lo sacara todo de encima - C18. Chico. Empresarios y profesionales - CFGS

Otros motivos que destacan para explicar su falta de identificación con la escuela y el desinterés que tenían es una alta exigencia del centro educativo:

Bueno, negativo en aquel momento era que fui a un instituto que creo que era bastante exigente - C21. Chico. Empresarios y profesionales Universidad

Y, finalmente, también destacan que una falta de interés por las materias y el contenido los llevó a no esforzarse en sus estudios:

No hacía lo que realmente me gustaba (...) Pues bueno hacía cosas que no me interesaban a mí, como en la ESO que me obligaban a hacer biología, pues a mí la biología nunca me ha llamado la atención. O la religión o no sé, la literatura... Quizá la literatura es más de cultura general pero nunca me llamaba la atención - C22. Chico. Empresarios y profesionales - Universidad

Lo único que claro, yo soy una persona vaga y el interés, mostraba más interés por las asignaturas que me gustaban y menos por las otras. Entonces, la profesora me decía en plan te tienes que esforzar y a ver que tengo buena experiencia con ella, pero me podría haber esforzado más y tal. - C15. Chico. Empresarios y profesionales - CFGM

A pesar de esto, muchos de ellos, al recordarlo pasados unos años lo valoran todo positivamente. Los jóvenes entrevistados hacen referencia a la edad, a la adolescencia e inmadurez que tenían. 


\section{Valls - Resistencias y Adhesiones Escolares}

En el momento sí que era que estaba como agobiado, pero al final se agradece cuando terminas y no sé, fueron buenos años, me lo pasé bien - C21. Chico. Empresarios y profesionales - Universidad

Ahora lo recuerdo bonito. Cuando estaba allí no, pero ahora que han pasado unos años, entonces ya si - C23. Chico. Empresarios y profesionales - Universidad

Bien, también te digo que no tenía la misma conciencia que tengo ahora. Yo ahora los miro de otra forma y los trato de otra forma. Ahora tengo el criterio para poder decir: tú vales y tú no vales como profesor. Antes, para mí, ninguno valía, pero era yo el que no valía - C17. Chico. Pequeños propietarios y trabajadores no manuales - CFGS

Por otro lado, vemos como varios de los chicos de clase media o mediabaja que tenían un peor rendimiento escolar y siguen itinerario de estudios profesionales tienen la universidad como objetivo. Esto nos permite confirmar nuestra tercera hipótesis dado que los chicos de clase media que desarrollan actitudes de resistencia son conscientes que independientemente de su actitud y comportamiento irán a la universidad. Un hecho que, como veremos, a partir de nuestras entrevistas no se observa en el caso de las chicas resistentes.

Yo tenía la ilusión de hacer alguna cosa relacionada con la sanidad y ya sabía de mis capacidades, lo que sí y lo que no. No podía hacer un bachillerato científico, sacar buena nota y entrar a enfermería o a cualquier cosa del estilo (...) Si lo que de verdad quiero hacer es llegar a la universidad y trabajar de eso, utiliza el grado superior para conseguir nota para llegar a eso - C17. Chico. Pequeños propietarios y trabajadores no manuales - CFGS

A mí el bachillerato no me gustaba y cuando me vinieron a dar una charla de los grados (FP) no lo vi con mala cara. Lo vi como una oportunidad de hacer alguna cosa que me gustaba y cuando descubrí que podía hacer el de deportes dije, para hacer un bachillerato que a mí no me gusta, hago un grado de deportes que a mí me gusta. (...) Me gustaría hacer INEF, pero al final lo que me gusta es estar con niños y quiero hacer magisterio, educación primaria, y después cuando termine 
la carrera universitaria hacer una carrera de psicología deportiva. - C18. Chico. Empresarios y profesionales - CFGS

Chicas resistentes. Es destacable el caso de las chicas resistentes que abandonan sus estudios. A diferencia de los chicos ambas presentaban actitudes anti-escuela muy marcadas:

En esa escuela eran muy "pijos" si no encajaba en su perfil era como que ya no les gustabas y a los profesores igual. Muchas cosas positivas no tengo, la verdad. Bueno... positivas, el temario estaba bien, supongo, o algo, pero...positivas tengo más recuerdos negativos la verdad. Porque salí muy quemada de esa escuela. - C13. Chica. Empresarios y profesionales - Abandono

En ambos casos exponen importantes problemas con el profesorado, llegando incluso a expulsiones por el hecho de falsificar notas:

Entonces si ella también nos trata de manera incorrecta, nosotros no teníamos tampoco, si ella nos trataba incorrectamente nosotros no íbamos a tratarla correctamente. Pues fue también los dos casos, tanto el alumno como el profesor (...) pienso que no fue un buen profesor (...) Por sus comportamientos en clase, el dirigirse hacia los alumnos, las formas de hacerlo - C14. Chica. Trabajadores manuales - Abandono

Por falsificar alguna nota si, por eso si que me expulsaron, pero por faltar a clases no. - C13. Chica. Empresarios y profesionales Abandono

Además, emerge el tema del absentismo escolar durante la educación secundaria obligatoria motivado por aburrimiento, influencia del grupo de pares o por desmotivación:

Uff siiii, en secundaria...Porque iba a repetir y, pues ya me lo pasé un poco, no iba a clase por la tarde. No iba muchas tardes - C13. Chica. Empresarios y profesionales - Abandono 


\section{Valls - Resistencias y Adhesiones Escolares}

Si. Por el motivo que me aburro en clase y me voy. Me voy en plan a los patios, a salir o directamente ya no vengo por la mañana. Por aburrimiento, a lo mejor había amigos que se quedaban y te ibas a la casa de uno o de otro. Te he dicho del camino bueno y malo... - C14. Chica. Trabajadores manuales - Abandono

Según su opinión había desinterés, indiferencia y unas bajas expectativas por parte del profesorado:

No, porque como iba a cambiar de escuela y al saber que tenía que cambiar de escuela les daba más o menos igual. Al final no me cambié - C14. Chica. Trabajadores manuales - Abandono

Me daban como por perdida, yo creo (...) Porque yo no les caía demasiado bien...a ver, no me comportaba muy bien yo tampoco ¿sabes? - C13. Chica. Empresarios y profesionales - Abandono

A pesar de todo, una de ellas, de clase baja, después de abandonar sus estudios considera que eligió un buen camino al haber conseguido el graduado de la ESO:

Lo viví en positivo porque al fin y al cabo supe por dónde ir ¿no? Esta es una etapa de muchos cambios y tienes un camino, dos caminos que elegir: el bueno y el malo, entonces depende de la persona. Entonces yo decidí por el bueno, también estuve a punto de ir por el malo. El malo yo creo que conlleva los suspensos, a repetir, a hacer cosas que no deberías, y...el bueno significa el hecho de sacarte la ESO, superarte a ti mismo, saber que si que puedes, aunque la gente dice que no. Y ... afrontar miedos - C14. Chica. Trabajadores manuales - Abandono

Finalmente, encontramos a otra joven que tiene actitudes de resistencia, pero en vez de abandonar sigue con estudios profesionales. En este caso su actitud se debía a desmotivación y dificultades. Cree que el profesorado era lejano, pero tiene buenos recuerdos del Instituto. Pero en ninguno de los tres casos entrevistados de chicas resistentes encontramos la motivación de estudiar para llegar a la universidad que sí que se ha observado en el caso de 
los chicos:

No me gustaba lo que hacía, pero nada. No me motivaba y siempre he tenido dificultades, por lo tanto, me fue mal - C19. Chica. Empresarios y profesionales - CFGS

\section{Discusión}

\section{Incidencia de los Factores Individuales y Sociales en las Actitudes Escolares}

Los resultados muestran, a partir de un enfoque interseccional, que el género, la clase social y el origen migratorio son factores que, tal y como apuntan otras investigaciones (Bonal et al., 2003), pueden condicionar el desarrollo de determinadas actitudes frente a la escuela. Respecto al género, según estudios anteriores (Bonal et al., 2003) y los resultados cuantitativos del estudio ISCY, las chicas tienen una mayor predisposición al estudio y una mejor adaptación al entorno escolar. Siendo los chicos los que tienen una mayor predisposición a la resistencia escolar (Gambetta, 1987). A pesar de esto en las entrevistas no se observan discursos diferenciados entre chicos y chicas a la hora de explicar sus actitudes. Solamente en el caso de las chicas resistentes entrevistadas, estas muestran un discurso anti-escuela más fuerte que los chicos resistentes. En futuras investigaciones se tendrá que profundizar en si hay formas de resistencia exclusivamente femenina como apuntan otras investigaciones (Griffin, 1985; McRobbie, 1978).

En el caso del origen migratorio, en consonancia con investigaciones anteriores (Rodríguez, 2015), emergen recuerdos y actitudes más positivas por parte del alumnado inmigrante que del alumnado autóctono. El alumnado de origen inmigrante se identifica instrumentalmente con la escuela dado que ve la educación como forma de ascenso social (Bonal et al., 2003), pero también se identifica expresivamente.

En referencia a la clase social, se observa que las actitudes de resistencia ya no se asocian principalmente con la clase trabajadora como apuntaban otros estudios. De este modo, los discursos de los chicos resistentes, mayoritariamente de clase media, se alinean más con los encontrados por 


\section{Valls - Resistencias y Adhesiones Escolares}

Aggleton (1987) o Anyon (1981) que encontraron resistencias en escuelas de clase media y alta a pesar de que no tenían un discurso claro de oposición. Y en el caso de los y las jóvenes entrevistados con actitud de adhesión son principalmente de clase baja y media baja.

Por otro lado, aspectos más vivenciales como acontecimientos críticos han afectado escolarmente a algunos de los jóvenes, como también apuntan otras investigaciones (García y Razeto, 2019; Subirats y Alegre, 2006). Principalmente los y las jóvenes de origen inmigrante, destacan situaciones personales y familiares complejas mostrando un gran sentimiento de superación durante la escolarización en el país de acogida. Por otro lado, también emerge la influencia de la edad y el efecto de la maduración personal en la identificación con la escuela (Duru-Bellat y Van Zanten, 1999; Pianta et al., 2012). Algunos jóvenes resistentes hacen referencia a la inmadurez durante la adolescencia para justificar su actitud, produciéndose un efecto de la maduración personal que en la postobligatoria ha modificado su identificación con la escuela.

\section{Contexto Escolar y Actitudes Frente a la Escuela}

En consonancia con estudios anteriores, del discurso de los y las entrevistados emerge la importancia del contexto institucional y las prácticas escolares a la hora de explicar su experiencia escolar y sus actitudes frente a la escuela (Ball, 1981; Bonal et al., 2010). De este modo, en el caso de los jóvenes resistentes destacan desmotivación y falta de interés por las materias y el contenido. También destacan una alta exigencia del centro educativo, aburrimiento y problemas y mala relación con el profesorado por el desinterés e indiferencia que mostraban hacia el alumnado. En cambio, el alumnado adherido destaca la acogida que tuvieron en el centro en el caso del alumnado de origen inmigrante, la ayuda y el apoyo del profesorado durante su proceso de aprendizaje y la ayuda en la obtención de la titulación de la ESO. De esta forma, como destacan otras investigaciones (Duru-Bellat y Van Zanten, 1999; García y Razeto, 2019), el profesorado juega un papel muy importante, tanto sus expectativas sobre el alumnado (Rist, 1991) como su acompañamiento. También las formas de acogida, capacidad de adaptación, y orientación son elementos clave para entender las actitudes. 
Algunos autores apuntan como un factor que impacta en la experiencia y vinculación del alumnado la agrupación escolar, pero el único tema que ha emergido en las entrevistas es el de la adaptación curricular. En este caso el alumnado entrevistado destaca positivamente esta adaptación dado que los ayudó a obtener el graduado de la ESO. Contrariamente a lo que apuntaban otras investigaciones (Bonal et al., 2010; Brown, 1987), los resultados de este estudio no muestran que unos malos resultados académicos lleven a actitudes de rechazo escolar. Ni unos buenos resultados a actitudes de adhesión (Brown, 1987; Duru-Bellat y Van Zanten, 1999; Everhart, 1983).

\section{Incidencia de las Actitudes Escolares en los Itinerarios Educativos}

Los resultados muestran que las actitudes escolares no tienen una incidencia clara sobre los itinerarios educativos. Como ya se observó en los resultados cuantitativos, son los factores sociales y familiares los que tienen una mayor incidencia en los itinerarios educativos que siguen los y las jóvenes. De este modo, a pesar de tener actitudes de adhesión y creer en la función de la escuela como mecanismo de movilidad social, como apuntaban otras investigaciones (Bonal et al. 2003; Suárez-Orozco y Suárez-Orozco 2008), muchos de los chicos entrevistados no pueden continuar sus estudios. En cambio, se observa que el hecho de desarrollar actitudes de adhesión beneficia positivamente a las chicas inmigrantes si tenemos en cuenta sus itinerarios formativos. Por otro lado, los jóvenes adheridos de origen autóctono, a pesar de que algunos tienen problemas de rendimiento, continúan con estudios postobligatorios.

En el caso de las actitudes de resistencia, varios de los chicos resistentes de clase media o media-baja que tenían un peor rendimiento escolar y siguen un itinerario de estudios profesionales tienen la universidad como objetivo. De este modo, los chicos de clase media que desarrollan actitudes de resistencia son conscientes que independientemente de su actitud y comportamiento irán a la universidad. Por lo tanto, las actitudes de resistencia no son un marcador de trayectorias futuras ni de fracaso escolar en el caso de los jóvenes de clase media. En cambio, las chicas resistentes entrevistadas presentan más conexión entre la actitud de resistencia y el abandono escolar independientemente de la clase social. 


\section{Valls - Resistencias y Adhesiones Escolares}

\section{Conclusiones}

Los resultados de esta investigación aportan conocimiento específico para profundizar en el debate de las actitudes del alumnado frente a la escuela desde un enfoque interseccional e incorporando las trayectorias educativas, sin focalizar exclusivamente en los itinerarios de abandono escolar. Por otro lado, el uso de métodos mixtos, escasamente utilizados en la literatura previa sobre el tema, ha permitido identificar las actitudes de adhesión y de resistencia escolar. Y, por otro lado, profundizar en el discurso de oposición o adhesión del alumnado que tenía estas actitudes.

La investigación muestra una interseccionalidad a la hora de analizar la actitud del alumnado al final de la educación obligatoria. Se observan importantes diferencias por clase, origen y género. Además, los resultados ponen de manifiesto el importante papel que juega el profesorado y el centro de enseñanza, sus prácticas y el acompañamiento que hacen del alumnado. Emergen como actores fundamentales en el acompañamiento del alumnado durante la educación secundaria obligatoria. En próximas investigaciones, a partir de una perspectiva de género, habrá que profundizar en los resultados encontrados. Se observan diferencias entre chicos y chicas, pero hay que incidir en los mecanismos que explican estas diferencias. De este modo, podremos explicar porque chicos y chicas presentando la misma actitud escolar en la ESO tienen trayectorias formativas diferenciadas, y si, por ejemplo, en el caso de las chicas de origen inmigrante y clase trabajadora con actitudes de adhesión lo que las lleva a estudiar a pesar de tener los mismo problemas económicos y familiares que los chicos es un mecanismo de compensación o de mayores oportunidades de inserción laboral. También será necesario profundizar en la influencia de otros elementos de la vinculación escolar sobre los itinerarios formativos de los y las jóvenes.

\section{Agradecimientos}

Plan Nacional I+D del Gobierno de España (referencia CSO2016-79945-P); Secretaria de Universidades e Investigación del Departamento de Empresa y Conocimiento de la Generalitat de Catalunya; Departamento de Sociología de la Universidad Autónoma de Barcelona. 


\section{Referencias}

Abraham, J. (1989). Testing Hargreaves' and Lacey's DifferentiationPolarisation Theory in a Setted Comprehensive. The British Journal of Sociology, 40(1), 46. https://doi.org/10.2307/590290

Aggleton, P. (1987). Rebels without a cause?: middle class youth and the transition from school to work. Falmer Press.

Alegre, M. À., \& Benito, R. (2012). Climes i (sobretot) cultures escolars: com s'expliquen i què permeten explicar. In $\mathrm{C}$. Gómez-Granell \& $\mathrm{P}$. Mari-Klose (Eds.), Família i relacions intergeneracionals: un espai d'oportunitats per a l'educació dels fills i filles (pp. 98-126).

Ajuntament de Barcelona - Institut d'Infància i Món Urbà. CIIMU. Anyon, J. (1981). Social Class and School Knowledge. Curriculum Inquiry, 11(1), 3. https://doi.org/10.2307/1179509

Archambault, I., Janosz, M., Fallu, J.-S., \& Pagani, L. S. (2009). Student engagement and its relationship with early high school dropout. Journal of Adolescence, 32, 651-670.

https://doi.org/10.1016/j.adolescence.2008.06.007

Ball, S. J. (1981). Beachside Comprehensive: a case study of secondary schooling. Cambridge University Press.

Barbeta, M., \& Termes, A. (2014). El rechazo escolar en diferentes contextos sociales: las resistencias y la reproducción de las contradicciones. Intersticios. Revista Sociológica de Pensamiento Crítico, 8(1). http://www.intersticios.es/article/view/12594

Bernstein, B. (1977). Class, Codes and Control. Vol. 3. Towards a Theory of Educational Transmission. Routledge y Kegan Paul.

Bonal, X., Alegre, M. A., González, I., Herrera, D., Rovira, M., \& Saurí, E. (2003). Apropiacions escolars. Usos i sentits de l'educació obligatòria en l'adolescencia (Fundació Jaumne Bofill (ed.)). Fundació Jaumne Bofill.

Bonal, X., Tarabini, A., Constans, M., Kliczkowski, F., \& Valiente, O. (2010). Ser pobre en la escuela. Habitus de pobreza y condiciones de educabilidad. Miño y Dávila.

Brown, P. (1987). Schooling ordinary kids : inequality, unemployment, and the new vocationalism. Tavistock Publications. 


\section{Valls - Resistencias y Adhesiones Escolares}

Carabaña, J. (1993). Sistema de enseñanza y clases sociales. In M. . A. García de Leon, G. de la Fuente, \& F. Ortega (Eds.), Sociología de la Educación. Barcanova.

Creswell, J. W., \& Guetterman, T. C. (2019). Educational research : planning, conducting, and evaluating quantitative and qualitative research (6th ed.). Pearson.

Dubet, F. (1992). Les acteurs du lycée. Informations Sociales, 18, 20-31. Duru-Bellat, M., \& Van Zanten, A. (1999). Sociologie de l'école. Armand Colin.

Erikson, R., \& Goldthorpe, J. H. (1992). The constant flux: Class mobility in industrial societies. Clarendon Press.

Everhart, R. B. (1983). Reading, writing, and resistance. Routledge \& Kegan Paul.

Galland, O. (1988). Représentation du devenir et reproduction sociale: le cas des lycéens d'Elbeuf. Sociologie Du Travail, 3, 399-417.

Gambetta, D. (1987). Were They Pushed or Did They Jump?: Individual Decision Mechanisms in Education. Cambridge University Press.

García Gracia, M., \& Razeto Pavez, A. (2019). ¿Por qué faltan los jóvenes a la escuela? Perfiles Educativos, 41(165), 43-61. https://doi.org/10.22201/iisue.24486167e.2019.165.59015

Griffin, C. (1985). Typical Girls?: Young Women from School to the Job Market. Routledge Kegan \& Paul.

Hamui-Sutton, A. (2013). Un acercamiento a los métodos mixtos de investigación en educación médica. Investigación En Educación Médica, 2(8), 211-216. https://doi.org/10.1016/s20075057(13)72714-5

Hargreaves, D. (1967). Social Relations in a Secondary School. Routledge \& Kegan Paul.

Macleod, F. (1995). Reading at Home: Does it Matter What Schools Do? Literacy, 29(3), 22-26. https://doi.org/10.1111/j.14679345.1995.tb00156.x

Martín-Criado, E., Gómez Bueno, C., \& Fernández Palomares, F. (2000). Éxito escolar y familias de clase obrera. In L. Samper (Ed.), Familia, cultura y educación (pp. 35-58). Servei de Publicacions de la Universitat de Lleida. 
McRobbie, A. (1978). Working Class Girls and the Culture of Femininity. In Women Take Issue. Hutchinson and the CCCS.

http://research.gold.ac.uk/14505/

Merton, R. K. (1938). Social Structure and Anomie. American Sociological Review, 3(5), 672-682. https://doi.org/10.4324/9781351157803-1

Pàmies, J., \& Castejón, A. (2015). Distribuyendo oportunidades: El impacto de los agrupamientos escolares en la experiencia de los estudiantes. RASE: Revista de La Asociación de Sociología de La Educación, 8(3), 335-348.

Ream, R. K., \& Rumberger, R. W. (2008). Student Engagement, Peer Social Capital, and School Dropout Among Mexican American and NonLatino White Students. Sociology of Education, 81, 109-139.

Rist, R. C. (1991). Sobre la comprensión del proceso de escolarización: aportaciones de la teoría del etiquetado. Educación y Sociedad, 9 , 179-191.

Rodríguez Izquierdo, R. M. (2015). Estudio de las actitudes hacia la escuela $\mathrm{y}$ de las expectativas educativas de los estudiantes de origen inmigrante. Education Policy Analysis Archives, 23, 127.

https://doi.org/10.14507/epaa.v23.2161

Suárez-Orozco, C., \& Suárez-Orozco, M. (2008). Històries d'immigració: la comprensió dels patrons de rendiment escolar dels joves immigrants nouvinguts. In Fundació Jaume Bofill.

Subirats, J. Alegre, C. Y. (2006). Convivencia social y convivencia escolar. Cuadernos de Pedagogía, 359, 12-16.

http://www.deciencias.net/convivir/2.protocolos/P.guias.convivir/Cua dernos_Pedagogia359/1.Contexto_y_sujeto/3.Convivencia_social(Joa n_Subirats).pdf

Toldson, I. A., McGee, T., \& Lemmons, B. P. (2013). Reducing Suspension among Academically Disengaged Black Males. In Howard University. https://doi.org/10.1017/CBO9781107415324.004

Valles, M. (1999). Técnicas cualitativas de investigación social. Síntesis. Valls, O. (2020) Attitudes, choices and inequalities in the transition from compulsory to post-compulsory school [Tesis doctoral]. Universitat Autònoma de Barcelona.

Wang, M.-T., Fredricks, J., Ye, F., Hofkens, T., \& Linn, J. S. (2019). 


\section{Valls - Resistencias y Adhesiones Escolares}

Conceptualization and Assessment of Adolescents' Engagement and Disengagement in School. European Journal of Psychological Assessment, 4(35), 592-606.

Willis, P. (1977). Learning to Labor: How Working Class Kids Get Working Class Jobs. Coger.

Woods, P. (1987). La escuela por dentro : la etnografía en la investigación educativa. Paidós.

Yang, M. Y., Chen, Z., Rhodes, J. L. F., \& Orooji, M. (2018). A longitudinal study on risk factors of grade retention among elementary school students using a multilevel analysis: Focusing on material hardship and lack of school engagement. Children and Youth Services Review, 88(February), 25-32. https://doi.org/10.1016/j.childyouth.2018.02.043

Ona Valls is a Postdoctoral Researcher at the Universität Wien, Austria

Contact Address: ona.valls@univie.ac.at 